\title{
BMJ Open Adaptive design methods in dialysis clinical trials: a systematic review protocol
}

\author{
Conor Judge (i) , ${ }^{1,2,3,4}$ Robert P Murphy (D) ,' Sarah Cormican, ${ }^{3,4}$ Andrew Smyth, ${ }^{1,4}$ \\ Martin O'Halloran, ${ }^{2}$ Martin O'Donnell ${ }^{1}$
}

\begin{abstract}
To cite: Judge C, Murphy RP, Cormican S, et al. Adaptive design methods in dialysis clinical trials: a systematic review protocol. BMJ Open 2020;10:e036755. doi:10.1136/ bmjopen-2019-036755
\end{abstract}

- Prepublication history and additional material for this paper are available online. To view these files, please visit the journal online ().

Received 02 January 2020 Revised 25 April 2020 Accepted 18 July 2020

\section{Check for updates}

(c) Author(s) (or their employer(s)) 2020. Re-use permitted under CC BY. Published by BMJ.

${ }^{1}$ HRB-Clinical Research Facility, National University of Ireland, Galway, Co. Galway, Ireland ${ }^{2}$ Translational Medical Device Lab, National University of Ireland Galway, Galway, Co. Galway, Ireland

${ }^{3}$ Wellcome Trust - HRB, Irish Clinical Academic Training, National University of Ireland Galway, Galway, Ireland ${ }^{4}$ Deparrtment of Nephrology, Galway University Hospital, Galway, Ireland

Correspondence to Dr Conor Judge; conor.judge@nuigalway.ie

\section{ABSTRACT}

Introduction Adaptive design methods are a potential solution to improve efficiency of clinical trials but their uptake in dialysis is unknown. We aim to investigate the use of adaptive design methods in dialysis clinical trials and to cultivate further adoption of adaptive design methods by the nephrology community.

Methods and analysis We will adhere to the Preferred Reporting Items for Systematic Review and Meta-Analysis Protocols guidelines and the Cochrane Collaboration Handbook. We will perform a literature search through MEDLINE (PubMed), EMBASE and CENTRAL, a detailed data extraction of trial characteristics and a narrative synthesis of the data. There will be no language restrictions. We will estimate the percentage of adaptive clinical trials per year in dialysis. Subgroup analysis will be performed by dialysis modality, funder and geographical location.

Ethics and dissemination Ethical approval will not be required for this study as data will be obtained from publicly available clinical trials. We will disseminate our results in a peer-reviewed publication. PROSPERO registration number

\section{INTRODUCTION}

\section{Background}

Randomised controlled trials (RCTs) are the gold standard for confirming efficacy or futility of new therapies. ${ }^{1}$ Nephrology as a specialty, and especially patients with endstage renal disease (ESRD), has traditionally had a low number of randomised trials compared with similar specialities. ${ }^{2}$ Many reasons have been cited for this including recruitment difficulty, history of underpowered trials and lack of funding. ${ }^{34}$ Nephrology studies are increasing in number but still lag behind other specialities. $^{5}$

There are an estimated 726331 prevalent cases of ESRD in the $\mathrm{USA}^{6}$ and globally, in 2010, an estimated 2.3-7.1 million people with end-stage kidney disease (ESKD) died without access to chronic dialysis. ${ }^{7}$ Projections estimate a $11 \%-18 \%$ increase in the crude incidence rate from 2015 to $2030 .{ }^{8}$ People with ESRD represent $1 \%$ of the Medicare

\section{Strengths and limitations of this study}

This study presents a comprehensive methodology for a systematic review of the current state of adaptive designs (ADs) clinical trials in dialysis.

- Two researchers will independently perform the study selection, data extraction and risk of bias assessment.

- Detailed characteristics of trials will be collected and analysed including nature of intervention, funding source, dialysis modality and the nature of the AD.

- Subgroup analysis will be performed to highlight differences in design characteristics between dialysis modality, temporal trends, types of intervention, funding and geographical location.

population but account for $7 \%$ of Medicare's expenditures. ${ }^{9}$ Additionally, people with ESRD experience higher mortality, ${ }^{10}$ morbidity and worse quality of life than the general public. ${ }^{11}$

Adaptive clinical trials use the results from interim data analysis of ongoing trials to modify the study design in a predefined way. ${ }^{12}$ This is performed without undermining the integrity or validity of the trial and thus preserving the type 1 error (false positive) rate. The most common type of adaptive design $(\mathrm{AD})$ is the Group Sequential Trial, where planned interim analysis enables stopping of trials for efficacy or futility. Other designs included sample size re-estimation, multiarm multistage (MAMS) trials, adaptive randomisation, biomarker adaptive and seamless phase II/III trials. ${ }^{13}$ Adaptive clinical trials would appear particularly suitable for evaluation of novel interventions in ESRD and according to the Food and Drug Administration, ADs could reduce resource requirements, decrease time to study completion and increase the likelihood of study success. ${ }^{14}$ A specific problem in previous ESRD trials includes over reliance on observational data in the design of clinical trials. ${ }^{15}$ Observational 
data are used to inform assumptions about the expected effect size and SD of effect size for power calculations. If these assumptions are incorrect, they can result in an underpowered trial with an insufficient sample size to answer the research question. Adaptive sample size re-estimation has been used successfully in cardiology trials to help this problem. ${ }^{16}$ Planned blinded sample size re-estimation can discover incorrect underlying assumptions and trigger increased recruitment targets mid trial to ensure adequate power to answer the research question. A second specific problem in previous ESRD trials is extrapolation of results from populations without Chronic Kidney Disease (CKD). An example of this was the 4D study, ${ }^{17}$ where $20 \mathrm{mg} /$ day of atorvastatin in patients with diabetes and ESRD did not reduce cardiovascular events, despite showing a $20 \%-30 \%$ reduction in populations without $\mathrm{CKD} .{ }^{18}$ One concern was that a single level of statin or a single low-density lipoprotein cut-off would not convey the same benefit in a ESKD population compared with a population without CKD. ${ }^{15}$ Adaptive MAMS trials have been successfully used in HIV trials, Telmisartan and Insulin Resistance in HIV used a MAMS design with one interim analysis to access three telmisartan doses (20, 40 and $80 \mathrm{mg}$ daily). ${ }^{19}$ MAMS designs are partially suited for populations with ESRD where, compared with populations without CKD, pharmacokinetic differences can be large and alternative drug doses are often required. ${ }^{20}$

\section{Objectives}

The systematic review will aim to: (1) summarise the use of $\mathrm{AD}$ methods in dialysis clinical trials per year, (2) describe the characteristics of the ADs including dialysis modality, funding and geographical location, (3) describe the AD characteristics of the trial, (4) estimate the percentage of adaptive clinical trials in dialysis and (5) outline trends in all the above.

\section{METHODS AND ANALYSIS}

This systematic review has been registered with PROSPERO (registration number: 163946 (Temporary ID)). We will conduct this systematic review according to the Preferred Reporting Items for Systematic Review and Meta-Analysis Protocols (PRISMA-P) and have completed the PRISMA-P checklist (online supplementary appendix $1)$.

\section{Inclusion/exclusion criteria for the selection of studies Type of study design and participants}

RCTs of interventions in patients with ESRD or acute kidney injury (AKI) undergoing renal replacement therapy (RRT) including haemodialysis, peritoneal dialysis, haemodiafiltration and haemofiltration. We will not limit our population or any specific disease.

\section{Type of intervention}

We will not place a restriction on the intervention type and will include trials that study medications during dialysis, medical devices, dialysis parameters, and so on.

\section{Type of outcome}

We will include all outcomes including surrogate markers, patient-centred outcomes and hard clinical outcomes.

\section{Search method for the identification of trials \\ Electronic search}

We will perform electronic searches on MEDLINE (PubMed), EMBASE and CENTRAL from database inception until 1 January 2020. Zotero will be used as our reference manager and the Revtools package on $\mathrm{R}$ will be used to eliminate duplicate records. The search will be conducted in English. The dialysis search terms were adapted fromBeaubien-Souligny $e t a l^{21}$ and include dialysis(tiab) OR peritoneal dialysis(tiab) OR hemodialysis(tiab) OR hemodiafiltration(tiab) OR haemodiafiltration(tiab) OR hemofiltration(tiab) OR haemofiltration OR extracorporeal blood cleansing(tiab) OR haemodialysis(tiab) OR Renal Dialysis (mh) OR Renal replacement(tiab) OR end stage kidney(tiab) OR end stage renal(tiab) OR stage five kidney(tiab) OR stage five renal(tiab).

The AD search terms were adapted from Bothwell et $a l^{22}$ and include 'phase ii/iii'(tiab) OR 'treatment switching'(tiab) OR 'biomarker adaptive'(tiab) OR 'biomarker adaptive design' (tiab) OR 'biomarker adjusted'(tiab) OR 'adaptive hypothesis'(tiab) OR 'adaptive dose-finding' (tiab) OR 'pick-thewinner' (tiab) OR 'dropthe-loser'(tiab) OR 'sample size re-estimation'(tiab) OR 're-estimations' (tiab) OR 'adaptive randomization'(tiab) OR 'group sequential' (tiab) OR 'adaptive seamless'(tiab) OR 'adaptive design' (tiab) OR 'Interim monitoring' (tiab) OR 'Bayesian adaptive'(tiab) OR 'Flexible design'”(tiab) OR 'Adaptive trial'(tiab) OR 'play-the-winner'(tiab) OR 'adaptive method'(tiab) OR (adaptive(All Fields) AND dose(All Fields) AND adjusting(All Fields)) OR 'response adaptive'(All Fields) OR 'adaptive allocation'(All Fields) OR 'adaptive signature design' (tiab) OR 'treatment adaptive' (tiab) OR 'covariate adaptive'(tiab) OR

'sample size adjustment'(tiab). We will perform two searches, first, we will combine the dialysis and adaptive search terms using a Boolean AND. The specific search will be carried out as described in table 1 . Second, we will perform a search with the dialysis search terms without the adaptive search terms for calculation of our total RCTs in dialysis denominator.

\section{Selection and analysis of trials}

We will use the high sensitivity machine learning classifier (RobotSearch) to identify RCTs from the combined search (dialysis and $\mathrm{AD}$ ) and from the dialysis search. ${ }^{23}$ RobotSearch is a machine learning classification algorithm combining an ensemble of support vector machines and convolutional neural networks with a reported area under the curve of 0.987 (95\% CI, 0.984 to 0.989 ). We 
Table 1 Search strategy for MEDLINE (PubMed)

dialysis[tiab]

OR

peritoneal dialysis(tiab)

OR

hemodialysis(tiab)

OR

hemodiafiltration(tiab)

OR

haemodiafiltration(tiab)

OR

hemofiltration(tiab)

OR

haemofiltration

OR

extracorporeal blood cleansing(tiab)

OR

haemodialysis(tiab)

OR

Renal Dialysis(mh)

OR

Renal replacement(tiab)

OR

end stage kidney(tiab)

OR

end stage renal(tiab)

OR

stage five kidney(tiab)

OR

stage five renal(tiab)
AND

OR

OR

treatment switching(tiab)

OR

biomarker adaptive(tiab)

OR

biomarker adaptive design(tiab)

OR

biomarker adjusted(tiab)

OR

adaptive hypothesis(tiab)

OR

adaptive dose-finding(tiab)

OR

pick-the winner(tiab)

OR

drop-the-loser(tiab)

OR

sample size re-estimation(tiab)

OR

re-estimations(tiab)

OR

adaptive randomization(tiab)

OR

group sequential(tiab)

OR

adaptive seamless(tiab)

OR

adaptive design(tiab)

OR

Interim monitoring(tiab)

OR

Bayesian adaptive(tiab)

OR

Flexible design(tiab)

OR

Adaptive trial(tiab)

OR

play-the-winner(tiab)

OR

adaptive method(tiab)

OR

(adaptive(All Fields) AND dose(All Fields) AND adjusting(All Fields)

OR

response adaptive(All Fields) 
Table 1 Continued

\begin{tabular}{ll} 
& OR \\
& adaptive allocation(All Fields) \\
OR & adaptive signature design(tiab) \\
& OR \\
& treatment adaptive(tiab) \\
& OR \\
& covariate adaptive(tiab) \\
& OR \\
& sample size adjustment(tiab). \\
\hline
\end{tabular}

will manually confirm a random sample $(10 \%)$ of studies classified as 'Not RCT' after the RobotSearch screening step. We will then review the title and abstracts of studies to confirm that they are RCTs and identify trials with $\mathrm{AD}$ methods for inclusion or exclusion. Studies with insufficient information to determine use of $\mathrm{AD}$ methods will also be included for full-text review. We will then perform full-text review to confirm studies that will be included in the final systematic review. This process will be summarised in a PRISMA flowchart. Abstract, title and full-text review will be performed by CJ and RPPM. Disagreements will be resolved by consensus or by a third reviewer $(\mathrm{SC})$, if necessary.

CJ and RPPM will extract the following information (adapted from Hatfield et al 2016) ${ }^{24}$ in parallel and record in a custom database (summarised in table 2):

Table 2 Characteristics of the trials

\section{Study characteristics Categories}

Nature of $A D$

\section{Stopping rule}

Year of study completion

Population under study None

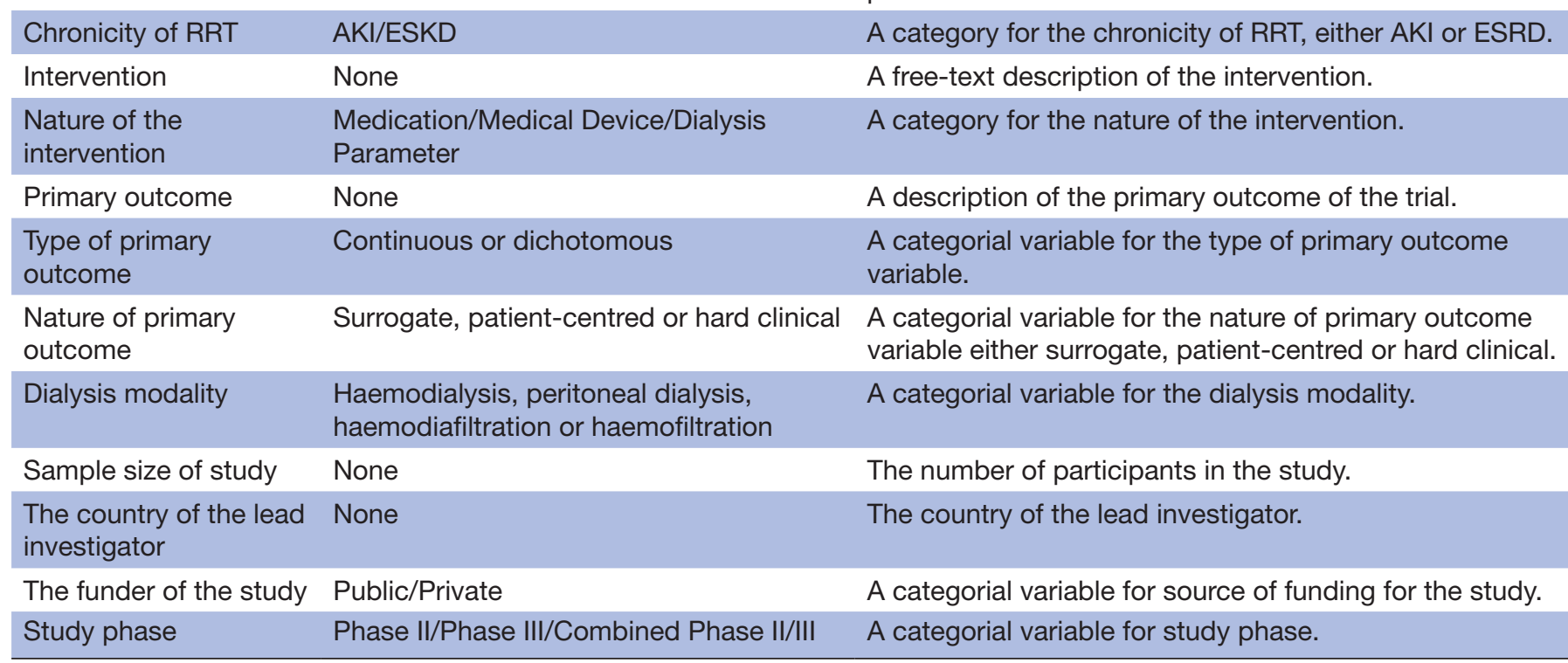

$A D$, adaptive design; AKI, acute kidney injury; ESKD, end-stage kidney disease; ESRD, end-stage renal disease; RRT, renal replacement therapy.

\section{Description}

Group Sequential Design (GSD) / Sample The type of AD used in the trial.

Size Re-estimation (SSR) / Dose Selection

(DS)/ Dose Escalation (DE) / Seamless / Interim Analysis

Futility/Efficacy/Two sided/N/A

If a stopping rule was used, what was the nature of the stopping rule.

The year of study completion.

A description of the population studied for example, patients with diabetes.

A category for the chronicity of RRT, either AKI or ESRD.

A free-text description of the intervention.

A description of the primary outcome of the trial.

A categorial variable for the type of primary outcome variable either surrogate, patient-centred or hard clinical.

The number of participants in the study.

A categorial variable for source of funding for the study. 
1. The type of the $\mathrm{AD}$ for example, dose-finding, adaptive hypothesis, group sequential, adaptive randomisation, seamless phase II/III, adaptive treatment-switching, biomarker adaptive and sample size re-estimation.

2. Stopping rule (futility or efficacy).

3. Year of completion of study.

4. Trial population.

5. Duration of time participants was receiving RRT (AKI or ESKD).

6. Intervention.

7. Domain of the intervention (medication, medical device or dialysis parameter).

8. Primary outcome measure.

9. Type of primary outcome variable (continuous or dichotomous).

10. Nature of primary outcome (surrogate outcomes, patient-reported measures or hard clinical outcomes).

11. Dialysis modality (haemodialysis, peritoneal dialysis, haemodiafiltration or haemofiltration).

12. Sample size.

13. The country of the lead investigator.

14. The funder of the study (public or private).

15. Published in journal with impact factor $>10$.

\section{Assessment of the quality of the studies: risk of bias}

We will use the Cochrane Risk of Bias Tool ${ }^{25}$ to assess methodological quality of eligible trials including random sequence generation, allocation concealment, blinding of participants and healthcare personnel, blinded outcome assessment, completeness of outcome data, evidence of selective reporting and other biases. Risk of bias assessments will be performed independently by two reviewers (SC, RPPM), and disagreements were resolved by a third reviewer $(\mathrm{CJ})$. If two of the domains was rated as high, the study was considered at high risk of bias. We will create a risk of bias summary table using Review Manager 5.3. ${ }^{26}$

\section{Data synthesis}

A descriptive synthesis of the data will be performed. We will estimate the percentage of adaptive clinical trials in dialysis per year by dividing the adaptive clinical trials per year by the total number of RCTs per year.

\section{Analysis by subgroups}

We will report overall outcomes and outcomes by dialysis modality (haemodialysis, peritoneal dialysis, haemodiafiltration and haemofiltration).

\section{Study status}

This systematic review will start in April 2020.

\section{Patient and public involvement}

There was no formal patient and public involvement in the design of this study.

\section{Ethics and dissemination}

Ethical approval was not required for this study. We will publish the results of this systematic review in a peerreviewed journal.

\section{DISCUSSION}

The number of clinical trials performed in ESRD is low compared with other medical subspecialties. ${ }^{27}$ Additionally, the cost of conducting RCTs are rising ${ }^{24}$ and there are reduced funding sources available, especially for nephrology. ${ }^{4}$ Adaptive clinical trials hold the potential to increase the efficiency and number of RCTs in ESRD and dialysis.

We expect to provide the following results: first, we will report on the proportion of RCTs in dialysis that include an $\mathrm{AD}$; second, we will outline the most popular type of $\mathrm{AD}$ in ESRD trials; third, we will report the main dialysis modalities where ADs are used and possibly highlight underutilisation in additional modalities; and fourth, we will report the geographical locations and funding pattern for trials using $\mathrm{AD}$ in dialysis.

Furthermore, the main impact from this systematic review will be increased awareness in the nephrology community of the potential benefit of using ADs in clinical trials. Having clear examples and use cases of successful $\mathrm{AD}$ will stimulate further use of these designs. Patients with ESKD are often excluded from RCTs, we urgently need novel design methods for investigating treatments in this underserved population. This systematic review will highlight $\mathrm{ADs}$ as one part of the solution to this problem. A secondary impact from this systematic review will be an up-to-date census of all RCTs in dialysis with a yearly count of trials. This information can be used to influence funders and policymakers about the importance of funding nephrology and especially ESKD research.

\section{Limitations}

This review will have potential limitations including publication and reporting bias. We will not be able to include studies with unpublished data and we will misclassify studies that do not have clear reporting of ADs in their methodology.

\section{Twitter Conor Judge @conorjudge}

Contributors CJ, RPM and MOD designed the study. All authors reviewed and approved the final version of the protocol. CJ is the guarantor of the review.

Funding This work was performed within the Irish Clinical Academic Training (ICAT) Programme, supported by the Wellcome Trust and the Health Research Board (Grant Number 203930/B/16/Z), the Health Service Executive, National Doctors Training and Planning and the Health and Social Care, Research and Development Division, Northern Ireland. The funding source had no role in the study design, analysis or writing of report.

Competing interests None declared.

Patient consent for publication Not required.

Provenance and peer review Not commissioned; externally peer reviewed.

Open access This is an open access article distributed in accordance with the Creative Commons Attribution 4.0 Unported (CC BY 4.0) license, which permits 
others to copy, redistribute, remix, transform and build upon this work for any purpose, provided the original work is properly cited, a link to the licence is given, and indication of whether changes were made. See: https://creativecommons.org/ licenses/by/4.0\%.

\section{ORCID iDs}

Conor Judge http://orcid.org/0000-0001-9473-2920

Robert P Murphy http://orcid.org/0000-0001-5446-4175

\section{REFERENCES}

1 Bothwell LE, Greene JA, Podolsky SH, et al. Assessing the gold standard - lessons from the history of RCTs. N Engl J Med Overseas Ed 2016;374:2175-81.

2 Kovesdy CP. Clinical trials in end-stage renal disease-priorities and challenges. Nephrol Dial Transplant 2019;34:1084-9.

3 Baigent C, Herrington WG, Coresh J, et al. Challenges in conducting clinical trials in nephrology: conclusions from a kidney DiseaseImproving global outcomes (KDIGO) controversies conference. Kidney Int 2017;92:297-305.

4 Bryan L, Ibrahim T, Zent R, et al. The kidney research predicament. J Am Soc Nephrol 2014;25:898-903.

5 Chatzimanouil MKT, Wilkens L, Anders H-J. Quantity and reporting quality of kidney research. JASN 2019;30:13-22.

6 United States renal data system: chapter 1: incidence, prevalence, patient characteristics, and treatment modalities, 2018. Available: http://www.usrds.org [Accessed 18 Nov 2019].

7 Liyanage T, Ninomiya T, Jha V, et al. Worldwide access to treatment for end-stage kidney disease: a systematic review. Lancet 2015;385:1975-82.

8 McCullough KP, Morgenstern H, Saran R, et al. Projecting ESRD incidence and prevalence in the United States through 2030. J Am Soc Nephrol 2019;30:127-35.

9 UNITED STATES RENAL DATA SYSTEM: Chapter 9: Healthcare Expenditures for Persons with ESRD [Internet]., 2018. Available: http://www.usrds.org [Accessed 18 Nov 2019].

10 Robinson BM, Zhang J, Morgenstern $\mathrm{H}$, et al. Worldwide, mortality risk is high soon after initiation of hemodialysis. Kidney Int 2014;85:158-65.

11 Wyld M, Morton RL, Hayen A, et al. A systematic review and metaanalysis of utility-based quality of life in chronic kidney disease treatments. PLoS Med 2012;9:e1001307.

12 Chow S-C, Chang M, Pong A. Statistical consideration of adaptive methods in clinical development. J Biopharm Stat 2005;15:575-91.

13 Pallmann P, Bedding AW, Choodari-Oskooei B, et al. Adaptive designs in clinical trials: why use them, and how to run and report them. BMC Med 2018;16.
14 U.S. Food and Drug Administration. Research C for DE and. Adaptive Design Clinical Trials for Drugs and Biologics [Internet], 2019. Available: http://www.fda.gov/regulatory-information/searchfda-guidance-documents/adaptive-design-clinical-trials-drugs-andbiologics [Accessed 18 Nov 2018].

15 Novak JE, Inrig JK, Patel UD, et al. Negative trials in nephrology: what can we learn? Kidney Int 2008;74:1121-7.

16 Chaitman BR, Pepine CJ, Parker JO, et al. Effects of ranolazine with atenolol, amlodipine, or diltiazem on exercise tolerance and angina frequency in patients with severe chronic angina: a randomized controlled trial. JAMA 2004;291:309.

17 Wanner C, Krane V, März W, et al. Atorvastatin in patients with type 2 diabetes mellitus undergoing hemodialysis. $N$ Engl $J$ Med 2005;353:238-48.

18 Cheung BMY, Lauder IJ, Lau C-P, et al. Meta-Analysis of large randomized controlled trials to evaluate the impact of statins on cardiovascular outcomes. Br J Clin Pharmacol 2004;57:640-51.

19 Pushpakom S, Kolamunnage-Dona R, Taylor C, et al. TAlLoR (TelmisArtan and InsuLin Resistance in Human Immunodeficiency Virus [HIV]): An Adaptive-design, Dose-ranging Phase llb Randomized Trial of Telmisartan for the Reduction of Insulin Resistance in HIV-positive Individuals on Combination Antiretrovira Therapy. Clin Infect Dis 2020;70:2062-72.

20 Velenosi TJ, Urquhart BL. Pharmacokinetic considerations in chronic kidney disease and patients requiring dialysis. Expert Opin Drug Metab Toxicol 2014;10:1131-43.

21 Beaubien-Souligny W, Kontar L, Blum D, et al. Meta-Analysis of randomized controlled trials using Tool-Assisted target weight adjustments in chronic dialysis patients. Kidney Int Rep 2019;4:1426-34.

22 Bothwell LE, Avorn J, Khan NF, et al. Adaptive design clinical trials: a review of the literature and ClinicalTrials.gov. BMJ Open 2018;8:e018320.

23 Marshall IJ, Noel-Storr A, Kuiper J, et al. Machine learning for identifying randomized controlled trials: an evaluation and practitioner's guide. Res Synth Methods 2018;9:602-14.

24 Hatfield I, Allison A, Flight L, et al. Adaptive designs undertaken in clinical research: a review of registered clinical trials. Trials 2016;17:150.

25 Higgins JP. Revised Cochrane risk-of-bias tool for randomized trials (rob 2). in RoB2 development group, 2019. Available: https:// sites.google.com/site/riskofbiastool/welcome/rob-2-0-tool/currentversion-of-rob-2

26 The Nordic Cochrane Centre, The Cochrane Collaboration. Review manager (RevMan). Copenhagen, 2014

27 Inrig JK, Califf RM, Tasneem A, et al. The landscape of clinical trials in nephrology: a systematic review of ClinicalTrials.gov. Am J Kidney Dis 2014;63:771-80. 